\title{
Atteinte coronarienne et syndrome néphrotique au cours du lupus systémique : à propos d'une observation
}

\section{Coronary involvement and nephrotic syndrome in systemic lupus: A case report}

Q. Moyon a b, M. Schmidt b, A. Hertig c, P. Fouret d, D. Vauthier-Brouzes e, Z. Amoura a, F. Cohen Aubart a

a

Service de médecine interne 2, Centre national de référence maladies systémiques rares, lupus et syndrome des anticorps antiphospholipides, hôpital de la Pitié-Salpêtrière, Sorbonne université, Assistance publique-Hôpitaux de Paris, 47-83, boulevard de 1'Hôpital, 75013 Paris, France

b

Service de réanimation médicale, hôpital de la Pitié-Salpêtrière, Sorbonne université, Assistance publique-Hôpitaux de Paris, 75013 Paris, France

c

Service d'urgences néphrologiques et transplantation rénale, hôpital Tenon, Sorbonne université, Assistance publique-Hôpitaux de Paris, 75020 Paris, France

d

Service d'anatomo-pathologie, hôpital de la Pitié-Salpêtrière, Sorbonne université, Assistance publique-Hôpitaux de Paris, 75013 Paris, France

e

Service de gynécologie-obstétrique, hôpital de la Pitié-Salpêtrière, Sorbonne université, Assistance publique-Hôpitaux de Paris, 75013 Paris, France 


\section{Résumé}

Introduction Les atteintes cardiaques au cours du lupus systémique ont des causes variées. Observation Une patiente de 29 ans présentait 3 mois après une grossesse une poussée de lupus systémique précédemment connu, avec un syndrome néphrotique, puis un choc cardiogénique nécessitant la pose d'une assistance cardiaque extra-corporelle. La dysfonction ventriculaire était liée à un infarctus myocardique massif par thrombose de l'artère interventriculaire antérieure, associée à un athérome sous-jacent. L'âge jeune et l'absence de douleur thoracique étaient peu évocateurs d'une atteinte coronarienne initialement. La thrombose coronarienne était probablement favorisée par le syndrome néphrotique, au cours duquel le risque thrombotique artériel est augmenté, et moins connu que le risque thrombotique veineux.

Conclusion Une atteinte coronarienne doit être systématiquement évoquée devant une dysfonction ventriculaire chez les patients lupiques, y compris lorsqu'ils sont jeunes et en l'absence de douleur thoracique. Le syndrome néphrotique doit être identifié comme un facteur de risque de thrombose artérielle. 


\section{Summary}

Introduction Heart failure during systemic lupus erythematosus has various causes.

Case report A 29-year-old female presented with a systemic lupus flare and nephrotic syndrome, followed by cardiogenic shock requiring extra-corporeal membranous oxygenation. Ventricular dysfunction was related to massive myocardial infarction due to an anterior interventricular artery thrombosis and underlying atheroma. The young age and absence of chest pain were not suggestive of coronary artery disease initially. Coronary thrombosis was probably favored by the nephrotic syndrome, in which the arterial thrombotic risk is increased.

Conclusion Coronary artery disease should be systematically evoked incase of ventricular dysfunction in patients with systemic lupus, including when they are young and in the absence of chest pain. Nephrotic syndrome should be identified as a risk factor for arterial thrombosis. 
Le lupus systémique est une pathologie auto-immune multi-systémique touchant avec prédilection les femmes jeunes. Les manifestations cardiaques survenant au cours du lupus systémique peuvent être liées à la maladie elle-même, à l'athérosclérose, et/ ou à des thromboses, survenant dans le cadre d'un syndrome des anticorps antiphopholipides (SAPL) ou non. Les atteintes cardiaques du lupus systémique sont dominées par les péricardites [1]. Les atteintes myocardiques sont plus rares, et les atteintes de l'endocarde sont souvent associées à une biologie antiphospholipide [2, 3].

Nous rapportons ici le cas d'une patiente de 29 ans présentant 3 mois après une grossesse une poussée de lupus avec un syndrome néphrotique, puis un choc cardiogénique nécessitant la pose d'une assistance cardiaque extra-corporelle, dont les facteurs étiologiques sont discutés.

\section{Observation}

Une femme de 26 ans, agent administratif, d'origine Européenne, non tabagique, recevait en 2014 un diagnostic de lupus systémique devant une polyarthrite, une péricardite, une neuropathie optique rétro bulbaire inflammatoire, des anticorps antinucléaires et anti-acide désoxyribonucléique (ADN) double brin positifs. Elle n'avait pas d'antécédent familial cardiovasculaire, ni de facteur de risque cardiovasculaire. La patiente est alors traitée par prednisone et hydroxychloroquine. En 2015, une biopsie rénale pour protéinurie persistante sans insuffisance rénale, montrait une glomérulonéphrite lupique de classe IV, traitée par corticothérapie et mycophénolate, l'hydroxychloroquine étant poursuivie. En 2016, une première grossesse, non programmée, menée sous corticoïdes, azathioprine (en relais du mycophénolate lors de la grossesse), et hydroxychloroquine, se compliquait d'une préeclampsie précoce sévère avec HELLP (Hemolysis, Elevated Liver enzymes, Low platelet count) syndrome, menant à une interruption de grossesse à 20 semaines d'aménorrhée (SA). 
La patiente était adressée en néphrologie puis en médecine interne dans les suites de cette grossesse. Le lupus était alors quiescent sous 10 milligrammes par jour de prednisone, hydroxychloroquine, et azathioprine. La pression artérielle était contrôlée, la créatininémie à $68 \mu \mathrm{mol} / \mathrm{L}$, la protéinurie était $<0,5$ grammes/24 heures, et le complément normal. La biologie anti-phospholipide (anticoagulant circulant de type lupique, anticorps anticardiolipines IgG et IgM, et anticorps antibeta2 glycoprotéine 1 IgG et $\operatorname{IgM}$ ) était négative, de même que le dosage de l'homocystéine et le reste du bilan de thrombophilie. Une grossesse était démarrée en fin d'année 2016, avec une fausse-couche spontanée précoce à 6 SA, puis une nouvelle grossesse en début d'année 2017, la patiente étant alors sous 5 milligrammes de prednisone, hydroxychloroquine, et 2 milligrammes/kg d'azathioprine. En raison d'une majoration de la protéinurie à $12 \mathrm{SA}$ à $1,3 \mathrm{~g} / \mathrm{g}$, une biopsie rénale par voie transjugulaire était réalisée, montrant une classe IV isolée, sans signe d'activité, avec 30\% de chronicité, sans classe V associée. Le traitement n'était pas modifié. La protéinurie se majorait en cours de grossesse, et finalement l'accouchement était déclenché à $37 \mathrm{SA}$ en raison de la protéinurie, d'un petit poids pour l'âge gestationnel avec stagnation de la croissance permettant la naissance d'un garçon de 2400 grammes. Un mois après la naissance, le lupus était quiescent cliniquement, la protéinurie à 1 gramme/24 heures, le complément normal. Le traitement par hydroxychloroquine, prednisone 10 milligrammes, et azathioprine était maintenu, et du ramipril 5 milligrammes était introduit. Le dosage d'hydroxychloroquine dans le sang était $>750 \mathrm{ng} / \mathrm{mL}$. La patiente était revue 3 mois plus tard pour une prise de 7 kilogrammes et des oedèmes installés sur 2 à 3 semaines. Cliniquement, il existait des crépitants bilatéraux et des signes d'insuffisance cardiaque droite. La patiente ne décrivait pas de douleur thoracique ni de symptomatologie d'angor. La pression artérielle était à 105/60 mmHg, le pouls à 95/minute. La créatininémie était à 300umol/L, le rapport protéinurie/ 
créatininurie à $12 \mathrm{~g} / \mathrm{g}$ et l'albuminémie à $19 \mathrm{~g} / \mathrm{L}$. L'hémogramme était normal en dehors d'une lymphopénie. La troponine était à 200ng/L (normale $<15 \mathrm{ng} / \mathrm{L}$ ) et le NT pro BNP était supérieur à $35000 \mathrm{ng} / \mathrm{L}$ (normale $<300 \mathrm{ng} / \mathrm{L}$ ). L'électrocardiogramme était en rythme sinusal avec des ondes T négatives en antérieur étendu. La radiographie thoracique était compatible avec une surcharge gauche. La patiente était transférée dans le service de Réanimation, et devant ce tableau de décompensation cardiaque et rénale, l'hypothèse alors privilégiée était celle d'une glomérulonéphrite lupique, possiblement associée à une myocardite, également d'origine lupique. L'échocardiographie cardiaque transthoracique confirmait une altération modérée de la fraction d'éjection ventriculaire gauche (FEVG) à 35\%, sans troubles de cinétique segmentaire. La prise en charge initiale reposait sur des perfusions intraveineuses de methylprednisolone (500 milligrammes par jour), une anticoagulation efficace par héparine non fractionnée en raison du syndrome néphrotique avec hypo-albuminémie profonde, et une déplétion par furosémide. L’imagerie par résonnance magnétique (IRM) myocardique confirmait l'altération de la FEVG, sans argument pour une pathologie inflammatoire ou ischémique. Une biopsie rénale par voie transjugulaire, pour laquelle l'anticoagulation était interrompue pendant 6 heures, était réalisée au second jour de la prise en charge en réanimation, et montrait une glomérulonéphrite lupique de classe IV avec $40 \%$ d'activité et 100\% de chronicité, sans classe V associée.

Dans la nuit suivant la biopsie, la situation s'aggravait brutalement avec un tableau de choc cardiogénique et un bas débit généralisé sans douleur thoracique pour lequel une assistance circulatoire de type Extra Corporeal Membranous Oxygenation (ECMO) veino-artérielle était mise en place en urgence. Au décours de la pose de l'ECMO, l'échographie cardiaque montrait une akinésie cardiaque à l'échocardiographie avec une FEVG $<15 \%$ et une intégrale temps vitesse sous aortique effondrée. Le dosage de troponine était à $40000 \mathrm{ng} / \mathrm{L}$ et 
finalement une coronarographie était réalisée, montrant une occlusion proximale de l'artère interventriculaire antérieure, qui était traitée par angioplastie avec pose de stent, et une sténose d'une artère marginale, qui était respectée. Les suites étaient marquées par l'absence de récupération de la FEVG. Une insuffisance rénale anurique nécessitait une épuration extrarénale, et finalement une double greffe cardiaque et rénale était réalisée en Janvier 2018. L'évolution était lentement favorable, malgré de multiples complications infectieuses notamment. L'histologie du cœur explanté montrait un infarctus du myocarde en cours d'organisation des parois antérieure et latérale du ventricule gauche et des deux tiers antérieurs du septum interventriculaire, des foyers de nécrose ischémique sous-endocardique de la paroi postérieure du ventricule gauche et de la paroi antérieure du ventricule droit, et des thromboses fibrino-cruoriques de l'apex des deux ventricules. L'examen des artères coronaires montrait un athérome coronarien avec sténose serrée avant un stent de l'interventriculaire antérieure qui renfermait une thrombose en cours d'organisation (Figure 1).

La cause du choc cardiogénique était finalement un syndrome coronarien aigu, survenant chez une patiente lupique sans autre facteur de risque cardio-vasculaire que le lupus systémique et la maladie rénale, dans un contexte de syndrome néphrotique avec hypo-albuminémie profonde, sans biologie anti-phospholipide. Le diagnostic d'atteinte coronarienne athéromateuse avait été différé probablement du fait de l'âge jeune de la patiente, de l'absence de douleur thoracique, et du contexte de poussée de la maladie de fond. Le syndrome néphrotique aigu avait possiblement joué un rôle dans la survenue de cette thrombose coronaire, mais une atteinte athéromateuse incontestable était démontrée par la coronarographie et plus tard l'analyse de l'explant. 


\section{Discussion}

Nous décrivons ici le cas d'une patiente de 29 ans qui présentait un choc cardiogénique causé par un infarctus myocardique, au cours d'une poussée du lupus systémique avec un syndrome néphrotique, survenant 3 mois après une grossesse, et après une courte fenêtre où l'anticoagulation avait été suspendue pour la biopsie rénale. Le diagnostic d'atteinte coronarienne était différé, et l'absence de récupération de la FEVG nécessitait une transplantation cardiaque, accompagnée d'une transplantation rénale compte tenu des dommages antérieurs causés par le lupus systémique.

Les dysfonctions ventriculaires gauches au cours du lupus systémique peuvent être de causes diverses : inflammatoires au cours des myocardites lupiques [4], ischémiques en cas d'atteinte coronarienne macrovasculaire ou d'atteinte microvasculaire associée à un syndrome des anticorps antiphospholipides [5], toxiques (cyclophosphamide, rarement 1'hydroxychloroquine) [6, 7], ou en lien avec des pathologies de rencontre. La dysfonction ventriculaire gauche de la patiente à l'entrée en réanimation ne semblait ni inflammatoire ni ischémique sur l'IRM cardiaque initiale. Le choc cardiogénique fut d'abord mis sur le compte d'une myocardite dans le cadre de la poussée lupique, et la coronarographie fut réalisée avec délai. Il est possible que l'interruption de l'anticoagulation pour la biopsie rénale, la perfusion de méthylprednisolone, et le syndrome néphrotique per se, aient favorisé la thrombose coronarienne. La biopsie rénale était toutefois indiquée pour comprendre et traiter le syndrome néphrotique aigu. Par ailleurs, la patiente n'était pas sous aspirine depuis la fin de la grossesse, en l'absence de biologie anti-phospholipide [8, 9].

Ici, l'hypoalbuminémie profonde causée par le syndrome néphrotique avait probablement contribué à la thrombose coronarienne. Le syndrome néphrotique est en effet un facteur de risque de thrombose veineuse [10]. Dans une étude sur 1300 patients ayant un syndrome 
néphrotique, l'albuminémie inférieure à 29 g/L (Hazard Ratio (HR) 9,6), la présence d'une glomérulonéphrite extra-membraneuse (HR 10,8), un débit de protéinurie $>8 \mathrm{~g} / 24$ heures (HR 2,6), et le sexe masculin (HR 2,4) étaient identifiés comme facteurs de risque indépendants de thrombose [11]. L'état pro-thrombotique observé au cours des syndromes néphrotiques pourrait être lié à la fuite urinaire de molécules anticoagulantes comme les protéines $\mathrm{C}$ et $\mathrm{S}$, l'antithrombine, ou de pro-fibrinolytiques comme le plasminogène. Il existe également une activation de l'hémostase primaire [10]. Le lien entre syndrome néphrotique et thromboses artérielles est moins étayé. Dans une étude sur 300 patients atteints de syndrome néphrotique, 43 cas de thrombose artérielle étaient observés, soit une incidence de 1,5\% par année de suivi, et jusqu'à 5\% dans l'année qui suit le diagnostic [12]. Toutefois, à l'inverse de la thrombose veineuse où le rapport protéinurie sur albuminémie élevé constitue le principal facteur de risque de thrombose veineuse, les thromboses artérielles semblent plus liées aux facteurs de risque cardio-vasculaires traditionnels.

Plus spécifiquement, les thromboses coronariennes ont été peu décrites au cours des syndrome néphrotiques [13, 14]. Entre 1987 et 2018, 24 cas ont été publiés, dont les détails sont présentés dans le Tableau 1. En plus de ces cas, une étude rétrospective cas-contrôles de 142 patients avec un syndrome néphrotique et autant de témoins décrivait 11 infarctus du myocarde survenus chez les patients avec syndrome néphrotique, et aucun chez les témoins. Cependant, les caractéristiques démographiques, cliniques (facteurs de risques cardiovasculaires), et biologiques (protéinurie et albuminémie), des patients de cette étude n'étaient pas détaillées [13]. Les 24 patients de la littérature ayant présenté une thrombose coronarienne au cours d'un syndrome néphrotique étaient 17 hommes et 7 femmes d'âge médian au diagnostic de l'infarctus myocardique de 30 ans (de 7 à 72). La néphropathie la plus représentée était la glomérulonéphrite extra-membraneuse (10/24, 42\%). Le débit médian 
du rapport protéinurie/créatininurie était de $8,1 \mathrm{~g} / \mathrm{g}$ [1,5-12 g/g], et l'albuminémie médiane était de 19,5 g/L [14-33g/L]. Des facteurs de risque cardiovasculaires associés, comme l'hypertension artérielle, le tabagisme ou une contraception œstro-progestative, étaient décrits dans 9 cas sur 24 (38\%), sans prendre en compte la dyslipidémie, laquelle était au moins liée au syndrome néphrotique.

Chez la patiente dont l'histoire est décrite ici, le syndrome néphrotique n'était pas à lui seul responsable de la thrombose coronarienne. L'analyse pathologique du cœur explanté confirmait les données angiographiques et montrait la présence d'un athérome coronarien. Les données sur le risque cardio-vasculaire au cours du lupus systémique sont nombreuses [15]. Les événements ischémiques causés par l'athérosclérose représentent une cause significative de morbidité et de mortalité au cours du lupus systémique, comme au cours de l'insuffisance rénale chronique en général $[16,17]$. Les études autopsiques ont démontré les premières une augmentation de le la prévalence de l'athérome au cours du lupus et furent suivies par de multiples études ayant décrit une association entre le lupus systémique et des démonstrations indirectes de la présence d'athérome, comme l'épaisseur intima-média, ou les calcifications coronariennes [18-21]. Une étude prospective observationnelle a montré un risque relatif d'infarctus myocardique multiplié par 10 au cours du lupus systémique, comparativement à des patients non lupiques, après ajustement sur les facteurs de risques cardiovasculaires traditionnels, en particulier le tabac $[22,23]$. Chez notre patiente, il est possible que le syndrome néphrotique, et l'interruption même temporaire de l'anticoagulation pour la biopsie rénale, aient favorisé la survenue de la thrombose coronarienne, mais la présence d'athérome a été attestée par l'angiographie et l'analyse de l'explant cardiaque.

Enfin, chez notre patiente, la survenue d'une poussée du lupus dans les 3 mois qui ont suivi l'accouchement est démonstrative de l'augmentation du risque de poussée, au cours de la 
grossesse, mais également dans les 3 mois qui suivent. Cette augmentation de risque de poussée en cours de grossesse a été confirmée dans plusieurs études, essentiellement au 3 ème trimestre et dans les 3 mois suivant l'accouchement [24]. L'hydroxychloroquine réduit ce risque significativement, et notre patiente avait été traitée pendant toute sa grossesse et le post partum avec une bonne adhésion au traitement, comme attesté par les dosages sanguins d'hydroxychloroquine.

L'observation décrite ici rappelle l'importance de ne pas négliger les autres causes que le lupus per se, lors de la survenue de manifestations cliniques susceptibles d'être attribuées à une poussée, notamment pour les manifestations cardiovasculaires, et les complications ischémiques liées à une athérosclérose. Ici, la thrombose coronarienne était probablement multifactorielle (athérome, risque thrombotique lié au syndrome néphrotique, voire perfusion de méthylprednisolone). Un diagnostic précoce d'atteinte coronarienne peut permettre de proposer un traitement adapté, en particulier une angioplastie précoce. Cette observation pousse à intégrer le syndrome néphrotique comme un sur-risque thrombotique artériel, moins connu que le risque thrombotique veineux. Cette observation illustre donc la nécessité d'être particulièrement vigilant sur le risque de thrombose coronarienne chez les patients lupiques néphrotiques. 
Figure 1. Analyse histologique de l'explant cardiaque

L'image montre un athérome coronarien avec sténose serrée avant un stent de

l'interventriculaire antérieure (non visualisé sur la coupe) qui renferme une thrombose en cours d'organisation. Coloration hematoxyline éosine safran, faible grossissement. 


\begin{tabular}{|c|c|c|c|c|c|c|}
\hline & Article & $\begin{array}{c}\hat{\text {Asge}} \\
\text {, } \\
\text { Sex } \\
\text { e }\end{array}$ & $\begin{array}{c}\text { Néphropat } \\
\text { hie }\end{array}$ & $\begin{array}{l}\text { Albumi } \\
\text { ne }(\mathrm{g} / \mathrm{L})\end{array}$ & $\begin{array}{l}\text { Protéinur } \\
\text { ie }(g / g)\end{array}$ & $\begin{array}{c}\text { Autres facteurs de } \\
\text { risque } \\
\text { cardiovasculaires }\end{array}$ \\
\hline 1 & Zeng 2018 [25] & $\begin{array}{l}22 \\
\mathbf{M}\end{array}$ & GNMP & 19 & 10 & Aucun \\
\hline 2 & & $\begin{array}{l}29 \\
\mathbf{M}\end{array}$ & GEM & ND & ND & Dyslipidémie \\
\hline 3 & & $\begin{array}{l}54 \\
\mathbf{M}\end{array}$ & GEM & 25 & ND & Aucun \\
\hline 4 & & $\begin{array}{l}65 \\
M\end{array}$ & GEM & 30 & ND & HTA, Tabac \\
\hline 5 & & $\begin{array}{l}\mathbf{5 9} \\
\mathbf{M}\end{array}$ & GEM & 18 & ND & HTA, Tabac \\
\hline 6 & xle $201 /[14]$ & $\begin{array}{l}\mathbf{5 8} \\
\mathbf{M}\end{array}$ & GEM & 20 & ND & Aucun \\
\hline 7 & & $\begin{array}{l}72 \\
\text { F }\end{array}$ & GEM & 33 & ND & HTA \\
\hline 8 & & $\begin{array}{l}62 \\
\mathbf{M}\end{array}$ & GEM & 26 & ND & HTA \\
\hline 9 & & $\begin{array}{l}\text { 41, } \\
\text { F }\end{array}$ & GEM & 16 & ND & Aucun \\
\hline $\begin{array}{l}1 \\
0\end{array}$ & Zhao 2017 [26] & $\begin{array}{l}15 \\
\text { F }\end{array}$ & GNMP & 24 & 10 & Aucun \\
\hline $\begin{array}{l}1 \\
1\end{array}$ & Chu 2017 [27] & $\begin{array}{l}26 \\
M\end{array}$ & GEM & ND & 2,1 & Aucun \\
\hline $\begin{array}{l}1 \\
2\end{array}$ & Krishna 2015 [28] & $\begin{array}{l}28 \\
\mathbf{M}\end{array}$ & GNMP & 19 & 1,5 & Aucun \\
\hline $\begin{array}{l}1 \\
3\end{array}$ & Khan 2012 [29] & $\begin{array}{l}13 \\
\mathbf{M}\end{array}$ & LGM & ND & ND & ND \\
\hline $\begin{array}{l}1 \\
4\end{array}$ & Karabay 2012 [30] & $\begin{array}{l}21 \\
\mathbf{M}\end{array}$ & Amylose & 19 & 4 & Aucun \\
\hline $\begin{array}{l}1 \\
5\end{array}$ & $\begin{array}{l}\text { Suryawanshi } 2011 \\
\text { [31] }\end{array}$ & $\begin{array}{l}12 \\
\mathrm{M}\end{array}$ & LGM & ND & ND & Aucun \\
\hline $\begin{array}{l}1 \\
6\end{array}$ & Bramstedt 2010 [32] & $\begin{array}{l}\text { 41, } \\
\text { F }\end{array}$ & LGM & 14 & 12 & $\begin{array}{c}\text { Contraception, } \\
\text { Tabac }\end{array}$ \\
\hline
\end{tabular}




\begin{tabular}{|c|c|c|c|c|c|c|}
\hline $\begin{array}{l}1 \\
7\end{array}$ & Silva $2002[33]$ & $\begin{array}{l}7, \\
\mathbf{M}\end{array}$ & HSF & 26 & 10 & Aucun \\
\hline $\begin{array}{l}1 \\
8\end{array}$ & Hirano 2001 [34] & $\begin{array}{l}51 \\
\mathbf{M}\end{array}$ & Diabète 2 & 21 & 5 & Diabète, HTA \\
\hline $\begin{array}{l}1 \\
9\end{array}$ & Meyer 1998 [35] & $\begin{array}{l}37, \\
\text { F }\end{array}$ & LGM & 14 & 13 & Aucun \\
\hline $\begin{array}{l}2 \\
0\end{array}$ & Singh 1998 [36] & $\begin{array}{l}31 \\
\mathbf{M}\end{array}$ & GEM & 16 & 6,4 & Tabac \\
\hline $\begin{array}{l}2 \\
1\end{array}$ & Fearon 1996 [37] & $\begin{array}{l}\text { 17, } \\
\text { F }\end{array}$ & Lupus & ND & ND & ND \\
\hline $\begin{array}{l}2 \\
2\end{array}$ & Hорр 1994 [38] & $\begin{array}{l}7, \\
\mathbf{M}\end{array}$ & LGM & ND & ND & None \\
\hline $\begin{array}{l}2 \\
3\end{array}$ & $\begin{array}{l}\text { (No authors) } 1994 \\
\text { [39] }\end{array}$ & $\begin{array}{l}52 \\
\text { F }\end{array}$ & LGM & 22 & 2,8 & Obésité \\
\hline $\begin{array}{l}2 \\
4\end{array}$ & Fujimura 1987 [40] & $\begin{array}{c}\text { ND, } \\
\mathbf{M}\end{array}$ & ND & ND & ND & ND \\
\hline
\end{tabular}

Tableau 1. Cas rapportés de thromboses coronariennes au cours du syndrome néphrotique

M masculin ; F féminin ; GNMP glomérulonéphrite membrano proliférative ; GEM glomérulonéphrite extra-membraneuse ; LGM lésions glomérulaires minimes ; HSF hyalinose segmentaire et focale ; HTA hypertension artérielle ; ND non disponible 


\section{References}

1. Doria A, Iaccarino L, Sarzi-Puttini P, Atzeni F, Turriel M, Petri M. Cardiac involvement in systemic lupus erythematosus. Lupus. 2005;14(9):683-6.

2. Appenzeller S, Pineau CA, Clarke AE. Acute lupus myocarditis: Clinical features and outcome. Lupus. 2011;20(9):981-8.

3. Ruiz D, Oates JC, Kamen DL. Antiphospholipid Antibodies and Heart Valve Disease in Systemic Lupus Erythematosus. The American journal of the medical sciences. 2018;355(3):293-8.

4. Thomas G, Cohen Aubart F, Chiche L, Haroche J, Hie M, Hervier B, et al. Lupus Myocarditis: Initial Presentation and Longterm Outcomes in a Multicentric Series of 29 Patients. J Rheumatol. 2017;44(1):24-32.

5. Urbanus RT, Siegerink B, Roest M, Rosendaal FR, de Groot PG, Algra A.

Antiphospholipid antibodies and risk of myocardial infarction and ischaemic stroke in young women in the RATIO study: a case-control study. Lancet Neurol. 2009;8(11):998-1005.

6. Lin CJ, Vader JM, Slade M, DiPersio JF, Westervelt P, Romee R. Cardiomyopathy in patients after posttransplant cyclophosphamide-based hematopoietic cell transplantation. Cancer. 2017;123(10):1800-9.

7. Tselios K, Gladman DD, Harvey P, Mak S, Chantal M, Butany J, et al. Hydroxychloroquine-Induced Cardiomyopathy in Systemic Lupus Erythematosus. J Clin Rheumatol. 2016;22(5):287-8.

8. de Moreuil C, Fauchais AL, Merviel P, Tremouilhac C, Le Moigne E, Pasquier E, et al. [Pre-eclampsia prevention in 2018 in general population and in lupic women: At the dawn of a personalized medicine?]. Rev Med Interne. 2018;39(12):935-41.

9. Arnaud L, Mathian A, Devilliers H, Ruffatti A, Tektonidou M, Forastiero R, et al. Patient-level analysis of five international cohorts further confirms the efficacy of aspirin for the primary prevention of thrombosis in patients with antiphospholipid antibodies.

Autoimmun Rev. 2015;14(3):192-200.

10. Loscalzo J. Venous thrombosis in the nephrotic syndrome. The New England journal of medicine. 2013;368(10):956-8.

11. Barbour SJ, Greenwald A, Djurdjev O, Levin A, Hladunewich MA, Nachman PH, et al. Disease-specific risk of venous thromboembolic events is increased in idiopathic glomerulonephritis. Kidney Int. 2012;81(2):190-5.

12. Mahmoodi BK, ten Kate MK, Waanders F, Veeger NJ, Brouwer JL, Vogt L, et al. High absolute risks and predictors of venous and arterial thromboembolic events in patients with nephrotic syndrome: results from a large retrospective cohort study. Circulation. 2008;117(2): 224-30.

13. Ordonez JD, Hiatt RA, Killebrew EJ, Fireman BH. The increased risk of coronary heart disease associated with nephrotic syndrome. Kidney Int. 1993;44(3):638-42.

14. Xie L, Tang Y, Liu J, He SQ, Li JH, Zhu Y, et al. Acute myocardial infarction in patients of nephrotic syndrome: a case series. J Geriatr Cardiol. 2017;14(7):481-4.

15. Giannelou M, Mavragani CP. Cardiovascular disease in systemic lupus erythematosus: A comprehensive update. J Autoimmun. 2017;82:1-12. 
16. Andrades C, Fuego C, Manrique-Arija S, Fernandez-Nebro A. Management of cardiovascular risk in systemic lupus erythematosus: a systematic review. Lupus.

2017;26(13):1407-19.

17. Go AS, Chertow GM, Fan D, McCulloch CE, Hsu CY. Chronic kidney disease and the risks of death, cardiovascular events, and hospitalization. The New England journal of medicine. 2004;351(13):1296-305.

18. Bulkley BH, Roberts WC. The heart in systemic lupus erythematosus and the changes induced in it by corticosteroid therapy. A study of 36 necropsy patients. The American journal of medicine. 1975;58(2):243-64.

19. Fukumoto S, Tsumagari T, Kinjo M, Tanaka K. Coronary atherosclerosis in patients with systemic lupus erythematosus at autopsy. Acta Pathol Jpn. 1987;37(1):1-9.

20. Tyrrell PN, Beyene J, Feldman BM, McCrindle BW, Silverman ED, Bradley TJ. Rheumatic disease and carotid intima-media thickness: a systematic review and metaanalysis. Arterioscler Thromb Vasc Biol. 2010;30(5):1014-26.

21. Mavrogeni S, Koutsogeorgopoulou L, Dimitroulas T, Markousis-Mavrogenis G, Kolovou G. Complementary role of cardiovascular imaging and laboratory indices in early detection of cardiovascular disease in systemic lupus erythematosus. Lupus. 2017;26(3): 227-36.

22. Esdaile JM, Abrahamowicz M, Grodzicky T, Li Y, Panaritis C, du Berger R, et al. Traditional Framingham risk factors fail to fully account for accelerated atherosclerosis in systemic lupus erythematosus. Arthritis and rheumatism. 2001;44(10):2331-7.

23. Urowitz MB, Ibanez D, Gladman DD. Atherosclerotic vascular events in a single large lupus cohort: prevalence and risk factors. J Rheumatol. 2007;34(1):70-5.

24. Eudy AM, Siega-Riz AM, Engel SM, Franceschini N, Howard AG, Clowse MEB, et al. Effect of pregnancy on disease flares in patients with systemic lupus erythematosus. Ann Rheum Dis. 2018;77(6):855-60.

25. Zeng J, Li J, Zhang J. Acute anterior myocardial infarction in a 22-year-old male nephrotic patient along with familial hyperlipidaemia. Cardiol Young. 2018;28(11):1348-52. 26. Zhao Y, Su W, Liu S, Huo Q, Zhang H. Acute Myocardial Infarction in a Young Girl with Nephrotic Syndrome: A Case Report and Literature Review. Can J Cardiol. 2017;33(7): 950 e15- e17.

27. Chu Z, Zhu H, Zhang B, Jiang L. Myocardial Infarction in a Young Man With Nephrotic Syndrome. Int Heart J. 2017;58(2):275-8.

28. Krishna K, Hiremath S, Lakade S, Davakhar S. Acute Myocardial Infarction in Nephrotic Syndrome. J Assoc Physicians India. 2015;63(11):67-8.

29. Khan JA, Masood T, Shamsi F. Nephrotic syndrome: a rare cause of acute coronary syndrome in a child. J Coll Physicians Surg Pak. 2012;22(2):123-5.

30. Karabay CY, Kocabay G, Kalayci A. Acute inferior myocardial infarction with nephrotic syndrome. Am J Emerg Med. 2012;30(1):260 e1-3.

31. Suryawanshi SP, Das B, Patnaik AN. Myocardial infarction in children: Two interesting cases. Ann Pediatr Cardiol. 2011;4(1):81-3.

32. Bramstedt J, Schroder J, Dissmann R. [41-year-old female patient with ST-elevation myocardial infarction and multiple arterial emboli]. Internist (Berl). 2010;51(7):902-8.

33. Silva JM, Oliveira EA, Marino VS, Oliveira JS, Torres RM, Ribeiro AL, et al. Premature acute myocardial infarction in a child with nephrotic syndrome. Pediatr Nephrol. 2002;17(3):169-72. 
34. Hirano H, Takao M, Nomoto J, Matsunaga A, Tsuchiya Y, Ideishi M, et al. A giant left ventricular thrombus in a patient with acute myocardial infarction--a case report. Angiology. 2001;52(6):429-32.

35. Meyer T, Schulze F, Grone HJ, Kreuzer H. Simultaneous manifestation of acute myocardial infarction and nephrotic syndrome. Clin Cardiol. 1998;21(7):519-22.

36. Singh NP, Anuradha S, Ranjan P, Rizvi SN. Myocardial infarction in a young man. Postgrad Med J. 1998;74(870):247-9.

37. Fearon WF, Cooke JP. Acute myocardial infarction in a young woman with systemic lupus erythematosus. Vasc Med. 1996;1(1):19-23.

38. Hopp L, Gilboa N, Kurland G, Weichler N, Orchard TJ. Acute myocardial infarction in a young boy with nephrotic syndrome: a case report and review of the literature. Pediatr Nephrol. 1994;8(3):290-4.

39. A 52-year-old female with nephrotic syndrome and ischaemic heart disease. Natl Med J India. 1994;7(3):136-9.

40. Fujimura O, Gulamhusein S. Acute myocardial infarction: thrombotic complications of nephrotic syndrome. Can J Cardiol. 1987;3(6):267-9. 